April 25, 2000

\title{
Chiral Schwinger models without gauge anomalies
}

\author{
Harald Grosse* and Edwin Langmann** \\ *,** Erwin Schrödinger Internat. Inst. for Mathematical Physics, A-1090 Wien, Austria \\ ${ }^{*}$ Institut für Theoretische Physik, Universität Wien, A-1090 Wien, Austria \\ ** Theoretical Physics, Royal Institute of Technology, S-100 44 Stockholm, Sweden
}

\begin{abstract}
We find a large class of quantum gauge models with massless fermions where the coupling to the gauge fields is not chirally symmetric and which nevertheless do not suffer from gauge anomalies. To be specific we study two dimensional Abelian models in the Hamiltonian framework which can be constructed and solved by standard techniques. The general model describes $N_{\mathrm{P}}$ photon fields and $N_{\mathrm{F}}$ flavors of Dirac fermions with $2 N_{\mathrm{F}} N_{\mathrm{P}}$ different coupling constants i.e. the chiral component of each fermion can be coupled to the gauge fields differently. We construct these models and find conditions so that no gauge anomaly appears. If these conditions hold it is possible to construct and solve the model explicitly, so that gauge- and Lorentz invariance are manifest.
\end{abstract}

\section{Introduction}

In this paper we discuss a large class of gauge theory models where the gauge fields are coupled to $N_{\mathrm{F}}$ Dirac fermions with different coupling constants (= charges) $e_{j, \sigma}^{\alpha}$ where the index $\sigma=+,-$ distinguishes the chiral components of the fermions, $j=1,2, \ldots, N_{\mathrm{F}}$ is a fermion flavor index, and $\alpha=1,2, \ldots N_{\mathrm{P}}$ labels different gauge fields. As we shall see, the existence of large gauge transformations implies that the charges have to be quantized i.e. $e_{j, \sigma}^{\alpha}=n_{j, \sigma}^{\alpha} e^{\alpha}$ (no summation) with $n_{j, \sigma}^{\alpha}$ integers. We say that such a model is chirally symmetric if for all $\alpha$ and $j, n_{j,+}^{\alpha}=n_{\pi(j),-}^{\alpha}$ for some permutation $\pi$. 
It is worth noting that the standard Schwinger model is obtained as the special case $N_{\mathrm{P}}=N_{\mathrm{F}}=1$ and $n_{+}=n_{-}$, and the chiral Schwinger model corresponds to $N_{\mathrm{P}}=N_{\mathrm{F}}=1$ and $n_{+}=1$ and $n_{-}=0$ (for review on previous work on these and similar models see [1]). The class of models with only one photon field $\left(N_{\mathrm{P}}=1\right)$ and coupling constants $e n_{j,+}$ and $e n_{j,-}$ to the right- and left handed chiral components of the fermions, respectively ( $j=1, \ldots, N_{\mathrm{F}}$, and the $n_{j, \sigma}$ are integers) was previously proposed and studied in [1]. For these models it is known that the gauge anomaly cancels whenever

$$
\sum_{j=1}^{N_{\mathrm{F}}}\left(n_{j,+}^{2}-n_{j,-}^{2}\right)=0 .
$$

The simplest non-trivial (i.e. chirally asymmetric) solution is for $N_{\mathrm{F}}=2, n_{j,+}=(3,4)$ and $n_{j,-}=(0,5)$, and therefore one sometimes refers to this model as the 3-4-5 model [1]. Similarly one can find non-trivial examples for all Pythagorean Triple i.e. integer solutions of $a^{2}+b^{2}=c^{2}$.

For the general class of models with $N_{\mathrm{F}}$ fermions and $N_{\mathrm{P}} \leq N_{\mathrm{F}}$ photons which we study we find the following conditions for gauge anomalies to be absent,

$$
\sum_{j=1}^{N_{\mathrm{F}}}\left(n_{j,+}^{\alpha} n_{j,+}^{\beta}-n_{j,-}^{\alpha} n_{j,-}^{\beta}\right)=0 \quad \forall \alpha, \beta=1, \ldots, N_{\mathrm{P}} .
$$

A simple non-trivial example is for $N_{\mathrm{F}}=N_{\mathrm{P}}=2, n_{j,+}^{1}=(3,4), n_{j,-}^{1}=(0,5), n_{j,+}^{2}=(-1,2)$, and $n_{j,-}^{2}=(2,1)$. To find solutions of these conditions for given $N_{\mathrm{P}}$ and as small $N_{\mathrm{F}}$ as possible seems to be an interesting generalization of the problem of finding Pythagorean Triples.

The study of the 3-4-5 model in Ref. [1] was in the path integral formalism. Here we use a Hamiltonian approach in the spirit of Ref. [6]. This approach allows a rigorous construction of these model in the Hamiltonian framework using the quasi-free representation of boson- and fermion field algebras [3, 4], and we also can solve the model using standard bosonization techniques [5]. Our general class of models (i.e. arbitrary $N_{\mathrm{F}}$ and $N_{\mathrm{P}}$ ) is quite complicated, and it is somewhat surprising that it is possible to find the solution in such an explicit manner as we do in this paper. It is also intriguing to see the importance of the no-anomaly conditions in Eq. (2) at several different, seemingly unrelated points of our construction and solution.

To simplify notation we explain our methods and computations in detail for the simplest case $N_{\mathrm{P}}=1$, and we are careful to do things such that the generalization to the case $N_{\mathrm{P}}>1$ is easy.

The plan of this paper is as follows. We first concentrate on the models with one photon field and an arbitrary number $N_{\mathrm{F}}$ of fermion fields. After a formal definition we summarize the rigorous construction of these model in our framework. Anomalies are a consequence of 
Schwinger terms which result from the normal ordering necessary to construct the fermion currents as well-defined operators on the Hilbert space of the model. Especially we compute the gauge anomalies from the commutators of the implementers of gauge transformations (= Gauss' law generators), and Eq. (11) is obtained as condition for a vanishing gauge anomaly. If and only if this latter condition holds a simple construction and solution of this model is obtained. As mentioned, these results for $N_{\mathrm{P}}=1$ are presented such that the generalization to our general class of models with $N_{\mathrm{P}}$ photons and $N_{\mathrm{F}}$ fermions is easy. The presentation of our results for this latter case are given in a rather short final paragraph.

\section{Notation}

Throughout this paper we consider Abelian gauge theories with massless fermions on two dimensional spacetime which is a cylinder, $\mathbb{R} \times S^{1}$, parametrized by coordinates $\left(x^{\mu}\right)=$ $\left(x^{0}, x^{1}\right)$ where $x^{0}=t \in \mathbb{R}(=$ time $)$ and $x^{1}=x \in[-L / 2, L / 2]$ (= spatial coordinate; $0<L<\infty$ is the spatial length). Our metric tensor is $\operatorname{diag}(1,-1)$. We use the Einstein summation convention only for spacetime indices $\mu, \nu=0,1$ but not for flavor- or spin indices.

\section{Formal definition of the model}

We now define in some detail the simplest non-trivial example for a chiral model without gauge anomalies. As described above, this model contains $N_{\mathrm{F}}$ flavors of Dirac fermion fields $\psi_{j, \sigma}, \bar{\psi}_{j, \sigma}$ and one Abelian gauge field $A_{\mu}$; here and in the following, $\sigma, \sigma^{\prime}=+,-$ are spin indices, and indices $j, j^{\prime}=1, \ldots, N_{\mathrm{F}}$ distinguish the different fermion flavors.

The model is formally defined by the Lagrangian

$$
\mathcal{L}=-\frac{1}{4} F_{\mu \nu} F^{\mu \nu}+\sum_{j=1}^{N_{\mathrm{F}}} \bar{\psi}_{j} \gamma^{\mu}\left[-\mathrm{i} \partial_{\mu}+e\left(n_{j,+} P_{+}+n_{j,-} P_{-}\right) A_{\mu}\right] \psi_{j}
$$

where $F_{\mu \nu}=\partial_{\mu} A_{\nu}-\partial_{\nu} A_{\mu}$, and

$$
P_{ \pm}=\frac{1}{2}\left(\mathbf{1} \pm \gamma_{3}\right)
$$

are chiral projections; here $\gamma^{\mu} \equiv\left(\gamma^{\mu}\right)_{\sigma, \sigma^{\prime}}$ are Dirac matrices which we take as $\gamma^{0}=\sigma_{1}$, $\gamma^{1}=-\mathrm{i} \sigma_{2}$, and $\gamma_{3}=\gamma^{0} \gamma^{1}=\sigma_{3}\left(\sigma_{1,2,3}\right.$ are the Pauli spin matrices as usual), and the real parameters $e_{j, \sigma}=e n_{j, \sigma}$ are coupling constants. The gauge group for this model is $\mathcal{G}=C^{\infty}\left(\mathbf{R} \times S^{1} ; \mathrm{U}(1)\right)$ (= smooth $\mathrm{U}(1)$-valued functions on spacetime), and the Lagrangian Eq. (3) is obviously invariant under the following gauge transformations,

$$
\begin{aligned}
& \psi_{j} \rightarrow\left(\mathrm{e}^{\mathrm{i} n_{j,+} \chi} P_{+}+\mathrm{e}^{\mathrm{i} n_{j,-} \chi} P_{-}\right) \psi_{j} \\
& \bar{\psi}_{j} \rightarrow \bar{\psi}_{j}\left(\mathrm{e}^{-\mathrm{i} n_{j,+} \chi} P_{-}+\mathrm{e}^{-\mathrm{i} n_{j,-} \chi} P_{+}\right) \\
& A_{\mu} \rightarrow A_{\mu}-\frac{1}{e} \partial_{\mu} \chi
\end{aligned}
$$


for all $\mathrm{e}^{\mathrm{i} \chi} \in \mathcal{G}$. Note that the existence of the large gauge transformation $\mathrm{e}^{\mathrm{i} \chi(x, t)}=\mathrm{e}^{\mathrm{i} x / L}$ forces us to require that the $n_{j, \sigma}$ are integers (otherwise the large gauge transformations cannot be implemented in our model): The charges of the different fermion flavors have to be quantized.

To motivate our construction of the model in the Hamiltonian framework below we recall some formulas from the formal canonical procedure; see e.g. [2]. From the action Eq. (3) one computes the canonical momenta for the various fields of the model and obtains the following canonical (anti-) commutator relations

$$
\begin{aligned}
\left\{\psi_{j, \sigma}(x), \psi_{j^{\prime}, \sigma^{\prime}}^{\dagger}(y)\right\} & =\delta_{\sigma, \sigma^{\prime}} \delta_{j, j^{\prime}} \delta(x-y) \\
{\left[E(x), A_{1}(y)\right] } & =-\mathrm{i} \delta(x-y)
\end{aligned}
$$

etc. where $\psi^{\dagger}=\bar{\psi} \gamma^{0}$ and $E=F_{01}$. Moreover, with the notation

$$
\begin{aligned}
H_{0} & =\sum_{j=1}^{N_{\mathrm{F}}} \int_{-L / 2}^{L / 2} \mathrm{~d} x \psi_{j}^{\dagger}(x) \gamma_{3}\left(-\mathrm{i} \partial_{1}\right) \psi_{j}(x) \\
J(x) & =\sum_{j=1}^{N_{\mathrm{F}}} \sum_{\sigma= \pm} n_{j, \sigma} \psi_{j}^{\dagger}(x) \gamma_{3} P_{\sigma} \psi_{j}(x) \\
\rho(x) & =\sum_{j=1}^{N_{\mathrm{F}}} \sum_{\sigma= \pm} n_{j, \sigma} \psi_{j}^{\dagger}(x) P_{\sigma} \psi_{j}(x)
\end{aligned}
$$

the resulting Hamiltonian for the model can be written as

$$
H=H_{0}+\int_{-L / 2}^{L / 2} \mathrm{~d} x\left(\frac{1}{2} E(x)^{2}+e A_{1}(x) J(x)\right)
$$

and has to be supplemented by the constraint (= Gauss' law)

$$
G(x)=-\partial_{1} E(x)+e \rho(x) \simeq 0 .
$$

We note in passing that we also have two Noether currents, a vector current $\left(J_{\mathrm{V}}^{\mu}\right)$ and an axial current $\left(J_{\mathrm{A}}^{\mu}\right)$. These currents are given by

$$
\begin{gathered}
J_{\mathrm{V}}^{0}=\rho, \quad J_{\mathrm{V}}^{1}=J \\
J_{\mathrm{A}}^{0}=J, \quad J_{\mathrm{A}}^{1}=-\rho
\end{gathered}
$$

and formally (i.e. prior to quantization) obey continuity equations, $\partial_{\mu} J_{\mathrm{V}}^{\mu}=\partial_{\mu} J_{\mathrm{A}}^{\mu}=0$. We also introduce more general kinds of fermion currents

$$
\rho_{j, \sigma}(x)=\psi_{j, \sigma}^{\dagger}(x) \psi_{j, \sigma}(x)
$$

which all are observables of interest for our model.

\footnotetext{
${ }^{1}\{a, b\}=a b+b a$ and $[a, b]=a b-b a$

${ }^{2}$ here and in the following we set $t=0$ and make explicit the dependence on the spatial coordinate only
} 


\section{Construction of the model}

We now outline a rigorous construction of this model using the representation theory of loop groups [3, 4]. This construction amounts to representing the (Fourier modes) of the field operators $\psi_{j, \sigma}^{(*)}, A_{1}$ and $E$, and the observable algebra of the model by closed operators on a Hilbert space $\mathcal{H}$ such that the Hamiltonian $H$ is represented by a self-adjoint operator on $\mathcal{H}$. In this construction it is crucial to establish gauge- and Lorentz invariance. As we shall see, this will lead us to the condition in Eq. (11).

The essential physical requirement determining the construction of the model and implying a non-trivial vacuum structure is positivity of the Hamiltonian $H$ on the physical Hilbert space. As is well-known, this forces one to use a non-trivial representation of the field operators of the model. The essential simplification in $(1+1)$ (and not possible in higher) dimensions is that one can use a quasi-free representation for the fermion field operators corresponding to "filling up the Dirac sea" associated with the free fermion Hamiltonian $H_{0}$, and for the photon operators one can use a naive boson representation. We now describe this representation in more detail.

In the following it is convenient to work in Fourier space. We first introduce some useful notation which is such that in all equations the limit $L \rightarrow \infty$ is obvious. The Fourier space for even (periodic) and odd functions is

$$
\Lambda^{*} \equiv\left\{p=\frac{2 \pi}{L} n \mid n \in \mathbf{Z}\right\} \quad \text { and } \quad \Lambda_{o d d}^{*} \equiv\left\{q=\frac{2 \pi}{L}\left(n+\frac{1}{2}\right) \mid n \in \mathbf{Z}\right\}
$$

respectively. For functions $\hat{f}$ on Fourier space we write $\hat{\int}_{\Lambda^{*}} \hat{\mathrm{d}} p \hat{f}(p) \equiv \sum_{p \in \Lambda^{*}} \frac{2 \pi}{L} \hat{f}(p)$ and similarly for $\Lambda_{o d d}^{*}$, and the corresponding $\delta$-function satisfying $\hat{\int}_{\Lambda^{*}} \hat{\mathrm{d}} p^{\prime} \hat{\delta}\left(p-p^{\prime}\right) \hat{f}\left(p^{\prime}\right)=\hat{f}(p)$ is $\hat{\delta}\left(p-p^{\prime}\right) \equiv \frac{L}{2 \pi} \delta_{p, p^{\prime}}$. Our conventions for the Fourier transformed operators are,

$$
\hat{\psi}_{j, \sigma}^{(\dagger)}(q)=\int_{-L / 2}^{L / 2} \frac{\mathrm{d} x}{\sqrt{2 \pi}} \psi_{j, \sigma}^{(\dagger)}(x) \mathrm{e}^{(+)} \mathrm{i} q x \quad\left(q \in \Lambda_{o d d}^{*}\right)
$$

(i.e. we use anti-periodic boundary conditions for the fermions),

$$
\hat{A}_{1}(p)=\int_{-L / 2}^{L / 2} \frac{\mathrm{d} x}{2 \pi} A_{1}(x) \mathrm{e}^{-\mathrm{i} p x} \quad\left(p \in \Lambda^{*}\right)
$$

and in the other cases

$$
\hat{Y}(p)=\int_{-L / 2}^{L / 2} \mathrm{~d} x Y(x) \mathrm{e}^{-\mathrm{i} p x} \quad\left(p \in \Lambda^{*}\right) \quad \text { for } Y=E, \rho, J, \rho_{j, \sigma} \text { etc. }
$$

Therefore the non-trivial canonical (anti-) commutator relations of the field operators become $\left[\hat{A}_{1}(p), \hat{E}\left(p^{\prime}\right)\right]=\mathrm{i} \hat{\delta}\left(p+p^{\prime}\right)$ and

$$
\begin{aligned}
& \left\{\hat{\psi}_{j, \sigma}(q), \hat{\psi}_{j^{\prime}, \sigma^{\prime}}^{\dagger}\left(q^{\prime}\right)\right\}=\delta_{\sigma, \sigma^{\prime}} \delta_{j, j^{\prime}} \hat{\delta}\left(q-q^{\prime}\right) \\
& \left\{\hat{\psi}_{j, \sigma}^{(*)}(q), \hat{\psi}_{j^{\prime}, \sigma^{\prime}}^{(*)}\left(q^{\prime}\right)\right\}=0 \quad \forall p, p^{\prime}, j, j^{\prime} .
\end{aligned}
$$


The model will be constructed in the full Hilbert space $\mathcal{H}=\mathcal{H}_{\text {Photon }} \otimes \mathcal{H}_{\text {Fermion }}$.

For $\mathcal{H}_{\text {Photon }}$ we take the Fock space generated by boson field operators $b^{\dagger}(p)$ and $b^{\dagger}(p)=$ $b(p)^{*}, p \in \Lambda^{*},(*$ is the Hilbert space adjoint) obeying the commutator relations

$$
\left[b(p), b^{\dagger}\left(p^{\prime}\right)\right]=\hat{\delta}\left(p-p^{\prime}\right), \quad\left[b(p), b\left(p^{\prime}\right)\right]=0 \quad \forall p, p^{\prime}
$$

and a normalized state $\Omega_{\mathrm{P}} \in \mathcal{H}_{\text {Photon }}$ such that

$$
b(p) \Omega_{\mathrm{P}}=0 \quad \forall p .
$$

We then set

$$
\hat{A}_{1}(p)=\frac{1}{s}\left(b(p)+b^{\dagger}(-p)\right) \quad \hat{E}(p)=-\frac{\mathrm{i} s}{2}\left(b(p)-b^{\dagger}(-p)\right)
$$

with a parameter $s$ to be determined later. We recall that these requirements fix the Hilbert space $\mathcal{H}_{\text {Photon }}$ completely. In this setting we now construct bilinears in the photon field using normal ordering $: \cdots$ : with respect to the state $\Omega_{\mathrm{P}}$, e.g. $: b(p) b^{\dagger}\left(p^{\prime}\right):=b^{\dagger}\left(p^{\prime}\right) b(p)$ for all $p, p^{\prime}$.

For $\mathcal{H}_{\text {Fermion }}$ we take the Fermion Fock space generated by operators $\hat{\psi}_{j, \sigma}(q)$ and $\hat{\psi}_{j, \sigma}^{\dagger}(q)=\hat{\psi}_{j, \sigma}(q)^{*}$ obeying the relations Eq. (15) and a normalized state $\Omega_{\mathrm{F}} \in \mathcal{H}_{\text {Fermion }}$ such that

$$
\hat{\psi}_{+, j}(q) \Omega_{\mathrm{F}}=\hat{\psi}_{-, j}^{*}(-q) \Omega_{\mathrm{F}}=0 \quad \forall q>0, \forall j .
$$

We note that the state $\Omega_{\mathrm{F}}$ characterizing this representation of the fermion field algebra can be interpreted as Dirac sea associated with the free fermion Hamiltonian $H_{0}$. The presence of this Dirac sea requires normal-ordering : $\cdots$ : of the Fermion bilinears such as $H_{0}=\sum_{j} \hat{\int}_{\Lambda_{\text {odd }}^{*}} \hat{\mathrm{d}} q: q \hat{\psi}_{j}^{\dagger}(q) \gamma_{3} \hat{\psi}_{j}(q):$ and $\hat{\rho}_{j, \sigma}(p)$. This modifies the naive commutator relations of these operators which follow from the canonical anti-commutator relations Eq. (15) as Schwinger terms show up, see e.g. [7, 8, 4. The relevant commutators for us are,

$$
\begin{aligned}
{\left[\hat{\rho}_{j, \sigma}(p), \hat{\rho}_{j^{\prime}, \sigma^{\prime}}\left(p^{\prime}\right)\right] } & =\sigma \delta_{\sigma, \sigma^{\prime}} \delta_{j, j^{\prime}} p \hat{\delta}\left(p+p^{\prime}\right) \\
{\left[H_{0}, \hat{\rho}_{j, \sigma}(p)\right] } & =\sigma p \hat{\rho}_{j, \sigma}(p) .
\end{aligned}
$$

We also note that

$$
\hat{\rho}_{j,+}(p) \Omega_{\mathrm{F}}=\hat{\rho}_{j,-}(-p) \Omega_{\mathrm{F}}=0 \quad \forall p>0, \forall j
$$

which together with (20) shows that the $\hat{\rho}_{j,+}(p)$ (resp. $\left.\hat{\rho}_{j,-}(p)\right)$ give a highest (resp. lowest) weight representation of the Heisenberg algebra.

The (Fourier transformed) Gauss' law operators are now well-defined on $\mathcal{H}$,

$$
\hat{G}(p)=-\mathrm{i} p \hat{E}(p)+e \hat{\rho}(p)
$$


with $\hat{\rho}(p)=\sum_{j, \sigma} n_{j, \sigma} \hat{\rho}_{j, \sigma}(p)$. To determine a (possible) gauge anomaly we compute the commutators of these operators and obtain (we use Eq. (20))

$$
\left[\hat{G}(p), \hat{G}\left(p^{\prime}\right)\right]=e^{2} p \hat{\delta}\left(p+p^{\prime}\right) \sum_{j=1}^{N_{\mathrm{F}}} \sum_{\sigma= \pm} \sigma n_{j, \sigma}^{2} .
$$

We see that no gauge anomaly occurs if and only if the condition Eq. (1) holds. In the following we assume that this is the case.

We now can give a precise meaning to the formal Hamiltonian $H$ in Eq. (8) as follows,

$$
H=H_{0}+\hat{\int}_{\Lambda^{*}} \hat{\mathrm{d}} p:\left(\frac{1}{4 \pi} \hat{E}(p) \hat{E}(-p)+e \hat{A}_{1}(p) \hat{J}(-p)+\pi M^{2} \hat{A}_{1}(p) \hat{A}_{1}(-p)\right): \quad .
$$

Similarly as in the Schwinger model we have added a photon mass term (= last term on the r.h.s. of Eq. (24)) to restore gauge invariance which otherwise would be spoiled by Schwinger terms. Note that our normalization is such that this term formally equals $\frac{1}{2} M^{2} \int_{-L / 2}^{L / 2} \mathrm{~d} x A_{1}(x)^{2}$, i.e. $M$ can be interpreted as photon mass. To determine the parameter $M^{2}$ we compute $[\hat{G}(p), H]$ and demand this to be always zero. By straightforward computation (we use Eq. (20)),

$$
M^{2}=\frac{e^{2}}{2 \pi} \sum_{j=1}^{N_{\mathrm{F}}} \sum_{\sigma= \pm} n_{j, \sigma}^{2}
$$

We finally have to fix the parameter $s$ in Eq. (18). We observe that $H$ in Eq. (24) has the form $H=H_{0, \mathrm{~F}}+H_{0, \mathrm{P}}+H_{\text {int }}$ where $H_{0, \mathrm{~F}}=H_{0}$ and

$$
H_{0, \mathrm{P}}=\hat{\int}_{\Lambda^{*}} \hat{\mathrm{d}} p:\left(\frac{1}{4 \pi} \hat{E}(p) \hat{E}(-p)+\pi M^{2} \hat{A}_{1}(p) \hat{A}_{1}(-p)\right):
$$

are the free fermion- and Photon Hamiltonians. We fix $s$ by requiring that $H_{0, \mathrm{P}}$ has the form $\hat{\int}_{\Lambda^{*}} \hat{\mathrm{d}} p \omega(p) b^{\dagger}(p) b(p)$. We thus obtain $s=2 \sqrt{\pi|M|}$ and $\omega(p)=|M|$. Then $H_{0, \mathrm{~F}}+H_{0, \mathrm{P}}$ obviously is defined as self-adjoint, positive operators on $\mathcal{H}$. Our results in this paper imply that also the interacting Hamiltonian $H$ is well-defined on $\mathcal{H}$ : the operator $H$ is self-adjoint and bounded from below on $\mathcal{H}$.

\section{Bosonization and gauge fixing I}

As in the standard Schwinger model we can rewrite the Hamiltonians of our models as boson Hamiltonians by using the so-called Kronig identity $\hat{H}_{0}=\frac{1}{2} \hat{\int}_{\Lambda^{*}} \hat{\mathrm{d}} p: \sum_{j, \sigma} \hat{\rho}_{j, \sigma}(p) \hat{\rho}_{j, \sigma}(-p)$ : 
where $: \cdots$ : denotes normal ordering of the fermion currents (see [州 for more details). We obtain

$$
H=\hat{\int}_{\Lambda^{*}} \hat{\mathrm{d}} q:\left(\frac{1}{4 \pi} \hat{E}(p) \hat{E}(-p)+\frac{1}{2} \sum_{j=1}^{N_{\mathrm{F}}} \sum_{\sigma= \pm} \tilde{\rho}_{j, \sigma}(p) \tilde{\rho}_{j, \sigma}(-p)\right):
$$

where we have defined

$$
\tilde{\rho}_{j, \sigma}(p)=\hat{\rho}_{j, \sigma}(p)+\sigma e n_{j, \sigma} \hat{A}_{1}(p)
$$

Note that $\left[\hat{G}(p), \tilde{\rho}_{j, \sigma}\left(p^{\prime}\right)\right]=0$ i.e. the operators $\tilde{\rho}_{j, \sigma}(p)$ all are gauge invariant and thus observables for our model.

The Hilbert space $\mathcal{H}$ which we have obtained still contains gauge equivalent states i.e. states related to each other by static gauge transformations. To obtain the physical Hilbert space $\mathcal{H}_{\text {phys }}$ for our models we have to go through a procedure called fixing the gauge in the physics literature. We will do it in two steps. The first step will be done in this paragraph and eliminates the 'small' gauge transformations (i.e. the $\mathrm{e}^{\mathrm{i} \chi} \in \mathcal{G}$ with $\chi$ smooth and periodic functions on space $S^{1}$ ). This step is easy since the resulting Hilbert space $\mathcal{H}_{\text {phys }}^{\prime}$ can be identified as the sub-Hilbert space of $\mathcal{H}$ which is annihilated by all the Gauss law operators. In a second step, which will be described in Paragraph 7 further below, we will account for large gauge transformations, too.

For the first step we recall that the only gauge invariant degree of freedom of the Photon field at fixed time is the holonomy $\int_{-L / 2}^{L / 2} \mathrm{~d} x A_{1}(x)$. Due to the absence of a gauge anomaly we can therefore impose the gauge condition

$$
\hat{A}_{1}(p)=\delta_{p, 0} Y
$$

and solve the Gauss' law $\hat{G}(p) \simeq 0$ (cf. Eq. (22)) as

$$
\hat{E}(p)=\frac{e \hat{\rho}(p)}{\mathrm{i} p} \quad \text { for } p \neq 0 .
$$

This determines all components of $\hat{E}$ except the one conjugate to $Y$ :

$$
\hat{E}(0)=\frac{L}{2 \pi \mathrm{i}} \frac{\partial}{\partial Y}
$$

After that we are left with the $(p=0)$-component of Gauss' law, viz.

$$
e Q_{\mathrm{V}} \simeq 0, \quad Q_{\mathrm{V}}=\hat{\rho}(0)=\sum_{j=1}^{N_{\mathrm{F}}} \sum_{\sigma= \pm} n_{j, \sigma} \hat{\rho}_{j, \sigma}(0) .
$$

We thus can identify the Hilbert space of states invariant under all small gauge transformations as $\mathcal{H}_{\text {phys }}^{\prime}=L^{2}(\mathbf{R}, \mathrm{d} Y) \otimes \mathcal{H}_{\text {Fermion }}^{\prime}$ where $\mathcal{H}_{\text {Fermion }}^{\prime}$ is the zero charge sector 
of the fermionic Fock space. Moreover, by inserting Eqs. (29)-(31), each gauge invariant operator on $\mathcal{H}$ becomes an operator on $\mathcal{H}_{\text {phys }}^{\prime}$. Especially the Hamiltonian becomes $H=\frac{2 \pi}{L} \sum_{p \geq 0}: h_{p}:$ where

$$
h_{0}=-\frac{1}{4 \pi}\left(\frac{L}{2 \pi}\right)^{2} \frac{\partial^{2}}{\partial Y^{2}}+\frac{1}{2} \sum_{j=1}^{N_{\mathrm{F}}} \sum_{\sigma= \pm}\left(\hat{\rho}_{j, \sigma}(0)+\sigma n_{j, \sigma} e Y\right)^{2}
$$

and

$$
: h_{p}:=:\left(\frac{e^{2}}{2 \pi p^{2}} \hat{\rho}(-p) \hat{\rho}(p)+\sum_{j=1}^{N_{\mathrm{F}}} \sum_{\sigma= \pm} \hat{\rho}_{j, \sigma}(-p) \hat{\rho}_{j, \sigma}(p)\right): \text {. }
$$

This completes the first step of the gauge fixing procedure. As mentioned we have not yet accounted for the existence of the large gauge transformations. We will come back to this in Paragraph 7 below.

\section{Solution of the model}

The operators $: h_{p}$ : obviously all commute with each other. We thus can diagonalize the Hamiltonian $H$ by diagonalizing each term $: h_{p}$ : separately.

We first consider $: h_{p}$ : with $p>0$. We introduce the boson operators

$$
c_{j}(p)= \begin{cases}\frac{1}{\sqrt{|p|}} \hat{\rho}_{j,+}(p) & \text { for } p>0 \\ \frac{1}{\sqrt{|p|}} \hat{\rho}_{j,-}(p) & \text { for } p<0\end{cases}
$$

and $c_{j}^{\dagger}(p)=c_{j}(p)^{*}=c_{j}(-p)$, so that $\left[c_{j}(p), c_{j^{\prime}}^{\dagger}\left(p^{\prime}\right)\right]=\frac{L}{2 \pi} \delta_{j, j^{\prime}} \hat{\delta}\left(p-p^{\prime}\right)$ and $c_{j}(p) \Omega=0$ for all $j, j^{\prime}$ and $p, p^{\prime} \neq 0$. We also find it convenient to define

$$
c_{j}(p)=c_{j}(p), \quad c_{N_{\mathrm{F}}+j}(p)=c_{j}^{\dagger}(p) \quad j=1, \ldots N_{\mathrm{F}},
$$

to fix $p>0$, and suppress the argument $p$ in the following. Then $\left[c_{j}, c_{j^{\prime}}^{\dagger}\right]=\frac{L}{2 \pi} \delta_{j, j^{\prime}} q_{j}$ where $q_{j}=1$ and $q_{N_{\mathrm{F}}+j}=-1$ for $j=1, \ldots, N_{\mathrm{F}}$.

We then can write $: h_{p}:$ in matrix notation as $: \mathbf{c}^{\dagger} \cdot \mathbf{h c}:$ where we defined $\mathbf{c}^{\dagger}=$ $\left(c_{1}^{\dagger}, \ldots, c_{2 N_{\mathrm{F}}}^{\dagger}\right)$ etc. and $\mathbf{h}$ is the $2 N_{\mathrm{F}} \times 2 N_{\mathrm{F}}$ matrix

$$
\mathbf{h}=p \mathbf{1}+\frac{e^{2}}{2 \pi p} \mathbf{n} \otimes \mathbf{n}^{T}
$$

where $\mathbf{n}^{T}=\left(n_{1}, \ldots, n_{2 N_{\mathrm{F}}}\right)$ with $n_{j}=n_{j,+}$ and $n_{N_{\mathrm{F}}+j}=n_{j,-}$ for $j=1, \ldots, N_{\mathrm{F}}$ (1 is the $2 N_{\mathrm{F}} \times 2 N_{\mathrm{F}}$ unit matrix here, and we use a standard tensor notation; the ${ }^{T}$ denotes the 
matrix transpose). Defining $h_{p}=\mathbf{c}^{\dagger} \cdot$ hc we get $: h_{p}:=h_{p}-<\Omega, h_{p} \Omega>$ with

$$
<\Omega, h_{p} \Omega>=\frac{L}{2 \pi} \sum_{j=1}^{N_{\mathrm{F}}}\left(p+\frac{e^{2}}{2 \pi p} n_{j,-}^{2}\right)=\frac{L}{2 \pi}\left(N_{\mathrm{F}}|p|+\frac{M^{2}}{2 p}\right)
$$

(we used Eqs. (25) and (11)).

We now can diagonalize $h_{p}$ by finding a boson Bogoliubov transformation $\mathbf{C}=\mathbf{U c}(\mathbf{U}$ some $2 N_{\mathrm{F}} \times 2 N_{\mathrm{F}}$ matrix) so that the operators $C_{j}$ obey the same relations as the $c_{j}$ and are such that $h_{p}=\mathbf{C}^{\dagger} \cdot \mathbf{D C}=\sum_{j=1}^{2 N_{\mathrm{F}}} \omega_{j} C_{j}^{\dagger} C_{j}$. It is easy to see that these two conditions are equivalent to

$$
\mathbf{U} \mathbf{q} \mathbf{U}^{*}=\mathbf{q} \quad \text { and } \quad\left(\mathbf{U}^{-1}\right)^{*} \mathbf{h} \mathbf{U}^{-1}=\mathbf{D}
$$

where $\mathbf{q}=\operatorname{diag}\left(q_{1}, \ldots, q_{2 N_{\mathrm{F}}}\right)$ and $\mathbf{D}=\operatorname{diag}\left(\omega_{1}, \ldots, \omega_{2 N_{\mathrm{F}}}\right)\left(^{*}\right.$ and ${ }^{-1}$ is the matrix adjungation and matrix inverse, respectively). To solve this somewhat unconventional diagonalization problem we note that Eq. (39) implies

$$
\mathbf{D}^{2}=(\mathbf{q D})^{2}=\mathbf{U}(\mathbf{q h})^{2} \mathbf{U}^{-1},
$$

and $\mathbf{U}$ is determined from this equation up to transformations $\mathbf{U} \rightarrow \mathbf{V} \mathbf{U}$ where $\mathbf{V}$ commutes with $\mathbf{D}^{2}$. We shall see that Eq. (40) corresponds to a standard diagonalization problem: the matrix $(\mathbf{q h})^{2}$ is self-adjoint and thus can be diagonalized by a unitary matrix $\tilde{\mathbf{U}}$. We thus can solve the problem in Eq. (39) by first determining $\tilde{\mathbf{U}}$ and then making the ansatz $\mathbf{U}=\mathbf{V} \tilde{\mathbf{U}}$ with $\mathbf{V}$ commuting with $\mathbf{D}^{2}$. From Eq. (39) we then get the following condition,

$$
\mathbf{V} \tilde{\mathbf{U}} \mathbf{q h} \tilde{\mathbf{U}}^{*} \mathbf{V}^{-1}=\mathbf{q D}
$$

which again is a standard diagonalization problem and will allow us to determine $\mathbf{V}$.

We now compute $\mathbf{D}^{2}$ using Eq. (40). We write $\mathbf{q h}=p \mathbf{q}+\frac{M^{2}}{p} \mathbf{e}_{N_{\mathrm{F}}+1} \otimes \mathbf{e}_{1}^{T}$ where we define $\mathbf{e}_{1}=\mathbf{n} /|\mathbf{n}|\left(|\mathbf{n}|=\sqrt{\mathbf{n}^{T} \cdot \mathbf{n}}\right.$ ) and $\mathbf{e}_{N_{\mathrm{F}}+1}:=\mathbf{q} \mathbf{e}_{1}$ (we used $|\mathbf{n}|=|\mathbf{q n}|$ and $\frac{e^{2}}{2 \pi}|\mathbf{n}|^{2}=M^{2}$ ). It is now crucial to observe that the condition Eq. (11) is equivalent to $\mathbf{n}^{T} \cdot \mathbf{q n}=0$ i.e. if there is no gauge anomaly the two vectors $\mathbf{e}_{1}$ and $\mathbf{e}_{N_{\mathrm{F}}+1}$ are orthonormal. Moreover, in this case we can extend these vectors to a complete orthonormal real basis $\left\{\mathbf{e}_{j}\right\}_{j=1}^{2 N_{\mathrm{F}}}$ in $\mathbf{R}^{2 N_{\mathrm{F}}}$ so that $\mathbf{q e}_{j}=\mathbf{e}_{N_{\mathrm{F}}+j}$ for $j=1, \ldots, N_{\mathrm{F}}$. We thus obtain

$$
(\mathbf{q h})^{2}=p^{2} \mathbf{1}+M^{2}\left(\mathbf{e}_{1} \otimes \mathbf{e}_{1}^{T}+\mathbf{e}_{N_{\mathrm{F}}+1} \otimes \mathbf{e}_{N_{\mathrm{F}}+1}^{T}\right)
$$

from which we can immediately read off $\tilde{\mathbf{U}}$ and the matrix elements of $\mathbf{D}^{2}$ : denoting as $\mathbf{E}_{j}$ the standard basis in $\mathbf{R}^{2 N_{\mathrm{F}}}$ (i.e. $\left.\left(\mathbf{E}_{j}\right)_{j^{\prime}}=\delta_{j, j^{\prime}}\right)$ we have

$$
\tilde{\mathbf{U}}=\sum_{j=1}^{2 N_{\mathrm{F}}} \mathbf{E}_{j} \otimes \mathbf{e}_{j}^{T}
$$


and $\omega_{j}^{2}=p^{2}+M^{2}$ and for $j=1, N_{\mathrm{F}}+1$ and $\omega_{j}^{2}=p^{2}$ otherwise. We then compute

$$
\tilde{\mathbf{U}} \mathbf{q h} \tilde{\mathbf{U}}^{*}=\sum_{j=1}^{N_{\mathrm{F}}}\left(\frac{M_{j}^{2}}{p} \mathbf{E}_{N_{\mathrm{F}}+j} \otimes \mathbf{E}_{j}^{T}+p \mathbf{E}_{N_{\mathrm{F}}+j} \otimes \mathbf{E}_{j}^{T}+p \mathbf{E}_{j} \otimes \mathbf{E}_{N_{\mathrm{F}}+j}^{T}\right)
$$

with $M_{1}=M$ and $M_{j \neq 1}=0$, which shows that we can determine $\mathbf{V}$ by diagonalizing $2 \times 2$ matrices of the form $\left(\begin{array}{cc}0 & p \\ p+\frac{M_{j}^{2}}{p} & 0\end{array}\right)$. We find

$$
\mathbf{V}=\sum_{j=1}^{2 N_{\mathrm{F}}} \mathbf{E}_{j} \otimes \mathbf{F}_{j}^{T}
$$

where

$$
\mathbf{F}_{j, N_{\mathrm{F}}+j}=\frac{p \mathbf{E}_{j} \pm \sqrt{p^{2}+M_{j}^{2}} \mathbf{E}_{N_{\mathrm{F}}+j}}{\sqrt{2 p^{2}+M_{j}^{2}}} .
$$

Thus

$$
\omega_{j}=-\omega_{N_{\mathrm{F}}+j}=\sqrt{p^{2}+M_{j}^{2}} \quad \text { for } j=1, \ldots, N_{\mathrm{F}} .
$$

We thus obtain

$$
h_{p}=\sum_{j=1}^{N_{\mathrm{F}}} \sqrt{p^{2}+M_{j}^{2}}\left(C_{j}(p)^{\dagger} C_{j}(p)+C_{j}(-p) C_{j}(-p)^{\dagger}\right)
$$

where we used $C_{N_{\mathrm{F}}+j}(p)=C_{j}^{\dagger}(-p)$. This completes the diagonalization of the operators $: h_{p}:$.

We are left to diagonalize $h_{0}$. We note that this is the part of the Hamiltonian which contains the operators $Y$ and $\hat{\rho}_{j, \sigma}(0)$ which are not invariant under the large gauge transformation $\mathrm{e}^{\mathrm{i} x / L}$ but transform as follows,

$$
\begin{aligned}
Y & \rightarrow Y-\frac{1}{e} \\
\hat{\rho}_{j, \sigma}(0) & \rightarrow \hat{\rho}_{j, \sigma}(0)+\sigma n_{j, \sigma} .
\end{aligned}
$$

It is therefore not immediately obvious that $h_{0}$ is indeed invariant under the large gauge transformations. To make this invariance manifest we note that the operator $Q_{\mathrm{A}}=$ $\sum_{j, \sigma} \sigma n_{j, \sigma} \hat{\rho}_{j, \sigma}(0)$ changes under the transformations Eq. (48) as $Q_{\mathrm{A}} \rightarrow Q_{\mathrm{A}}+|\mathbf{n}|^{2}$, and therefore

$$
\tilde{Y}:=Y+\frac{1}{e|\mathbf{n}|^{2}} Q_{\mathrm{A}}
$$


is indeed invariant. By straightforward computations we obtain

$$
h_{0}=\frac{|M|}{2}\left(C_{1}(0)^{\dagger} C_{1}(0)+C_{1}(0) C_{1}(0)^{\dagger}\right)+\mathcal{C}
$$

where $C_{1}^{(\dagger)}(0)=\stackrel{(-)}{+} \frac{L}{4 \pi}(\pi|M|)^{-1 / 2} \frac{\partial}{\partial \tilde{Y}}+(\pi|M|)^{1 / 2} \tilde{Y}$ and

$$
\mathcal{C}=\frac{1}{2}\left(\sum_{j=1}^{N_{\mathrm{F}}} \sum_{\sigma= \pm} \hat{\rho}_{j, \sigma}(0)^{2}-\frac{1}{|\mathbf{n}|^{2}} Q_{\mathrm{A}}^{2}\right)
$$

all are invariant under the transformations Eq. (48). We also have $\left[C_{1}(0), C_{1}^{\dagger}(0)\right]=\frac{L}{2 \pi}$ and thus see that $h_{0}$ is essentially a harmonic oscillator Hamiltonian.

We can combine our results above in a compact form as follows,

$$
H=\sum_{j=1}^{N_{\mathrm{F}}} \hat{\int_{\Lambda^{*}}} \hat{\mathrm{d}} p \sqrt{p^{2}+M_{j}^{2}} C_{j}^{\dagger}(p) C_{j}(p)+\frac{2 \pi}{L} \mathcal{C}+\mathcal{E}_{0},
$$

with the constant

$$
\mathcal{E}_{0}=\sum_{j=1}^{N_{\mathrm{P}}}\left(\frac{\left|M_{j}\right|}{2}+\frac{L}{2 \pi} \hat{\int}_{p>0} \hat{\mathrm{d}} p\left(\sqrt{p^{2}+M_{j}^{2}}-\left(p+\frac{M_{j}^{2}}{2 p}\right)\right)\right)
$$

(note that actually only the term with $j=1$ contributes to $\mathcal{E}_{0}$ ). This shows explicitely that the Hamiltonian $H$ is self-adjoint and bounded from below, and since $\mathcal{C}$ is non-negative (we will show this further below) the constant $\mathcal{E}_{0}$ is equal to the the ground state energy. Moreover, we also see explicitly that our model has a relativistic spectrum: the physical degrees of freedom correspond to one massive- and $N_{\mathrm{F}}-1$ massless boson fields.

We now briefly describe how to construct a groundstate for our model. We note that with the explicit formulas given above it is straightforward to construct a unitary operator $\mathcal{U}(p)$ in terms of the operators $c_{j}^{(\dagger)}(p)$ such that $C_{j}(p)=\mathcal{U}(p) c_{j}(p) \mathcal{U}(p)^{*}$. Moreover, one can check that $\mathcal{U}=\prod_{p>0} \mathcal{U}(p)$ defines a unitary operator on $\mathcal{H}_{\text {Fermion }}^{\prime}$. It is then easy to see that the state $\varphi_{0}(Y) \mathcal{U} \Omega_{\mathrm{F}} \in \mathcal{H}_{\text {phys }}^{\prime}$ with $\varphi_{0}(Y) \propto \exp \left(-\pi|M| \frac{2 \pi}{L} Y^{2}\right)$ is annihilated by all operators $C_{j}(p)$ and $\mathcal{C}$ and thus a ground state of $H$. We thus have found one groundstate for our model. However, this state is highly degenerate, and it is actually not invariant under the large gauge transformations Eq. (48). In the next paragraph we will discuss this issue in more detail.

We finally mention that in a complete solution of the model one also needs to find the Green's functions i.e. vacuum expectation values of gauge invariant combinations of the fermion- and photon field operators. All these Green's functions can be computed explicitly by using the so-called boson-fermion correspondence which allows to write the fermion field operators in terms of the fermion currents. These computations are similar to the ones for the usual Schwinger model (see e.g. 泡) but beyond the scope of the present paper. 


\section{Gauge fixing II: Vacuum structure and all that}

We now describe the structure of the Hilbert space of our model and then perform the second step of the gauge fixing procedure described already above.

We first recall the well-known structure of the fermion Fock space $\mathcal{H}_{\text {Fermion }}$ : this space can be generated by the fermion current $\hat{\rho}_{j, \sigma}(p), p \neq 0$, together with unitary operators $R_{j, \sigma}$ which obey the relations

$$
R_{j, \sigma}^{-1} \hat{\rho}_{j^{\prime}, \sigma^{\prime}}(p) R_{j, \sigma}=\hat{\rho}_{j^{\prime}, \sigma^{\prime}}(p)+\delta_{p, 0} \delta_{j, j^{\prime}} \sigma \delta_{\sigma, \sigma^{\prime}}
$$

and which interpolate between different sectors labeled by the eigenvalues of the charge operators. Thus for all $\mathbf{m}=\left(m_{1,+}, \ldots, m_{N_{\mathrm{F}},+}, m_{1,-}, \ldots, m_{N_{\mathrm{F}},-}\right) \in \mathbf{Z}^{2 N_{\mathrm{F}}}$, the operators

$$
\mathbf{R}^{\mathbf{m}}=R_{1,+}^{m_{1,+}} \cdots R_{N_{\mathrm{F}},+}^{m_{N_{\mathrm{F}}+}} R_{1,-}^{m_{1,-}} \cdots R_{N_{\mathrm{F}},-}^{m_{N_{\mathrm{F}},-}}
$$

commute with all $h_{p>0}$ and $\mathcal{U}$, and if $\Psi$ is a vector in $\mathcal{H}_{\text {Fermion with }} \hat{\rho}_{j, \sigma}(0) \Psi=0 \forall j, \sigma$ then $\hat{\rho}_{j, \sigma}(0) \mathbf{R}^{\mathbf{m}} \Psi=m_{j, \sigma} \mathbf{R}^{\mathbf{m}} \Psi$, which implies that $\mathbf{R}^{\mathbf{m}} \Psi$ is an eigenvector of $Q_{\mathrm{V}}$ and $Q_{\mathrm{A}}$ with eigenvalues $\mathbf{n}^{T} \cdot \mathbf{m}$ and $\mathbf{n}^{T} \cdot \mathbf{q m}$, respectively. This implies that if such a vector $\Psi$ is also an eigenstate of all $h_{p>0}$ and $\varphi \in L^{2}(\mathbf{R})$ an eigenstate of $h_{0}$ then all states

$$
\varphi(\tilde{Y}) \mathbf{R}^{\mathbf{m}} \Psi=\varphi\left(Y+\frac{\mathbf{n} \cdot \mathbf{q m}}{e|\mathbf{n}|^{2}}\right) \mathbf{R}^{\mathbf{m}} \Psi
$$

with $\mathbf{n}^{T} \cdot \mathbf{m}=0$ (= charge zero condition) are eigenstates of $H$ with eigenvalues which are of the form $\mathcal{E}+c(\mathbf{m})$ and differ only by the contribution from $\mathcal{C}$ Eq. (51), $c(\mathbf{m})=$ $\frac{2 \pi}{L|\mathbf{n}|^{2}}\left(|\mathbf{m}|^{2}|\mathbf{n}|^{2}-\left(\mathbf{n}^{T} \cdot \mathbf{q m}\right)^{2}\right)$. Note that the latter is always non-negative due to the CauchySchwartz inequality. We thus see that all these states with $\mathbf{m}=k \mathbf{q n}$ ( $k$ integer) are degenerate, and especially all states $\varphi_{0}\left(Y+\frac{k}{e}\right) \mathbf{R}^{k \mathbf{q n}} \mathcal{U} \Omega$ are groundstates for our models. This degeneracy actually is explained by the invariance under large gauge transformation Eq. (48): This transformation acts on states in $\mathcal{H}_{\text {phys }}^{\prime}$ as $\varphi(Y) \Psi \rightarrow \varphi\left(Y+\frac{1}{e}\right) \mathbf{R}^{\mathbf{q n}} \Psi$.

We now come to the second step of our gauge fixing procedure. Our discussion above implies that the states

$$
[\Psi, \varphi]^{\theta}(Y)=\frac{1}{\sqrt{2 \pi}} \sum_{k \in \mathbf{Z}} \mathrm{e}^{\mathrm{i} k \theta} \varphi\left(Y+\frac{k}{e}\right) \mathbf{R}^{k \mathbf{q n}} \Psi
$$

( $\theta$ real) have simple transformation properties under large gauge transformation Eq. (48), $[\Psi, \varphi]^{\theta} \rightarrow \mathrm{e}^{\mathrm{i} \theta}[\Psi, \varphi]^{\theta}$. We thus can define $\mathcal{H}_{\text {phys }}$ as the vector space spanned by all $[\Psi, \varphi]^{0}(Y)$. However, this does not yet make $\mathcal{H}_{\text {phys }}$ into a Hilbert space: a simple computation shows that

$$
<\left[\Psi_{1}, \varphi_{1}\right]^{\theta},\left[\Psi_{2}, \varphi_{2}\right]^{\theta^{\prime}}>_{\mathcal{H}_{\text {phys }}}=\delta\left(\theta-\theta^{\prime}\right)<\left[\Psi_{1}, \varphi_{1}\right],\left[\Psi_{2}, \varphi_{2}\right]>_{\text {phys }}
$$


where $<\left[\Psi_{1}, \varphi_{1}\right],\left[\Psi_{2}, \varphi_{2}\right]>_{\text {phys }}=<\Psi_{1}, \Psi_{2}>_{\mathcal{H}_{\text {Fermion }}}<\varphi_{1}, \varphi_{2}>_{L^{2}(\mathbf{R})}$ is independent of $\theta$ and $\theta^{\prime}$. We see that the vectors $[\Psi, \varphi]^{\theta}(Y)$ are actually not contained in $\mathcal{H}_{\text {phys }}^{\prime}$ (they do not have finite norm). The remedy of this problem is a simple multiplicative regularization i.e. 'dropping the infinite constant $\delta(0)$ '. This is equivalent to using $<\cdot, \cdot\rangle_{\text {phys }}$ as inner product in $\mathcal{H}_{\text {phys }}$ which is well-defined. This completes the construction of the model.

\section{Generalization to an arbitrary number of photons}

We now describe how the above results generalize to a large class of models with $N_{\mathrm{P}}$ different photon fields $A_{\mu}^{\alpha}$ where $\alpha=1, \ldots, N_{\mathrm{P}}$. These models are (formally) given by ,

$$
\mathcal{L}=-\frac{1}{4} \sum_{\alpha=1}^{N_{\mathrm{P}}} F_{\mu \nu}^{\alpha}\left(F^{\alpha}\right)^{\mu \nu}+\sum_{j=1}^{N_{\mathrm{F}}} \bar{\psi}_{j} \gamma^{\mu}\left[-\mathrm{i} \partial_{\mu}+\sum_{\alpha=1}^{N_{\mathrm{P}}} e^{\alpha}\left(n_{j,+}^{\alpha} P_{+}+n_{j,-}^{\alpha} P_{-}\right) A_{\mu}^{\alpha}\right] \psi_{j}
$$

where $F_{\mu \nu}^{\alpha}=\partial_{\mu} A_{\nu}^{\alpha}-\partial_{\nu} A_{\mu}^{\alpha}$ and the charge units $e^{\alpha}$ corresponding to the different gauge fields can be different. This model obviously is invariant under transformations belonging to the gauge group $\mathcal{G}=C^{\infty}\left(\mathbf{R} \times S^{1} ; \mathrm{U}(1)^{N_{\mathrm{P}}}\right), A_{\mu}^{\alpha} \rightarrow A_{\mu}^{\alpha}-\frac{1}{e^{\alpha}} \partial_{\mu} \chi^{\alpha}$ etc., and as before the existence of large gauge transformations requires all the $n_{j, \sigma}^{\alpha}$ to be integers. The canonical procedure and the construction of the models with $N_{\mathrm{P}}=1$ generalize with minor changes: now we have $N_{\mathrm{P}}$ copies of the photon fields and correspondingly $N_{\mathrm{P}}$ copies of the Gauss' law operators etc. To be more specific: We now have $\left[\hat{A}_{1}^{\alpha}(p), \hat{E}^{\beta}\left(p^{\prime}\right)\right]=\mathrm{i} \delta^{\alpha, \beta} \hat{\delta}\left(p+p^{\prime}\right)$, a Hamiltonian

$H=H_{0}+\hat{\int}_{\Lambda^{*}} \hat{\mathrm{d}} p \sum_{\alpha=1}^{N_{\mathrm{P}}}:\left(\frac{1}{4 \pi} \hat{E}^{\alpha}(p) \hat{E}^{\alpha}(-p)+e^{\alpha} \hat{A}_{1}^{\alpha}(p) \hat{J}^{\alpha}(-p)+\pi \hat{A}_{1}^{\alpha}(p) \sum_{\beta=1}^{N_{\mathrm{P}}} \Upsilon^{\alpha \beta} \hat{A}_{1}^{\beta}(-p)\right):$,

and Gauss' law operators $\hat{G}^{\alpha}(p)=-i p \hat{E}^{\alpha}(p)+\hat{\rho}^{\alpha}(p)$ where

$$
\hat{\rho}^{\alpha}(p)=\sum_{j, \sigma} n_{j, \sigma}^{\alpha} \hat{\rho}_{j, \sigma}(p), \quad \hat{J}^{\alpha}(p)=\sum_{j, \sigma} \sigma n_{j, \sigma}^{\alpha} \hat{\rho}_{j, \sigma}(p)
$$

with $\hat{\rho}_{j, \sigma}(p)$ as before. The model has no gauge anomalies if and only if all commutators $\left[\hat{G}^{\alpha}(p), \hat{G}^{\beta}\left(p^{\prime}\right)\right]$ vanish, and similarly as for $N_{\mathrm{P}}=1$ we obtain the conditions in Eq. (2). Note that the mass term we have to add to the naive Hamiltonian depends on a $N_{\mathrm{P}} \times N_{\mathrm{P}}$-matrix $\Upsilon^{\alpha \beta}$ which is determined such that $H$ commutes with all $\hat{G}^{\alpha}(p)$. We thus obtain

$$
\Upsilon^{\alpha \beta}=\frac{e^{\alpha} e^{\beta}}{2 \pi} \sum_{j=1}^{N_{\mathrm{F}}} \sum_{\sigma= \pm} n_{j, \sigma}^{\alpha} n_{j, \sigma}^{\beta} .
$$


We note already here that $\Upsilon=\left(\Upsilon^{\alpha \beta}\right)_{\alpha, \beta=1}^{N_{\mathrm{P}}}$ is a self-adjoint, real, non-negative $N_{\mathrm{P}} \times N_{\mathrm{P}}$ matrix, and therefore we can write

$$
\Upsilon^{\alpha \beta}=\sum_{\gamma=1}^{N_{\mathrm{P}}} M_{\gamma}^{2}\left(a_{\gamma}\right)^{\alpha}\left(a_{\gamma}\right)^{\beta}
$$

where $\left(a_{\gamma}\right)^{\alpha}$ are the components of the orthonormal eigenvectors of $\Upsilon$ and $M_{\gamma}^{2}$ the corresponding eigenvalues. For later convenience we assume that $\operatorname{rank}(\boldsymbol{\Upsilon})=N_{\mathrm{P}}$ i.e. that all the $M_{\gamma}^{2}$ are non-zero. ${ }^{3}$

The generalization of the representation Eq. (18) of the photon fields etc. generalizes in a straightforward manner (we only mention that the generalization of the free Photon Hamiltonian now becomes $H_{0, \mathrm{P}}=\sum_{\alpha} \hat{\int}_{\Lambda^{*}} \hat{\mathrm{d}} p\left|M_{\alpha}\right| b_{\alpha}^{\dagger}(p) b_{\alpha}(p)$ where $M_{\alpha}^{2}$ are the eigenvalues of the matrix $\mathbf{\Upsilon})$. Moreover, also the generalization of Eq. (27) is obvious where the gauge invariant currents now are $\tilde{\rho}_{j, \sigma}(p)=\hat{\rho}_{j, \sigma}(p)+\sigma \sum_{\alpha=1}^{N_{\mathrm{P}}} e^{\alpha} n_{j, \sigma}^{\alpha} \hat{A}_{1}^{\alpha}(p)$ (note that due to Eq. (2) these latter currents indeed commute with all Gauss law operators).

We now come to the solution of the models: the gauge fixing condition generalizing the one in Eq. (29) obviously is $\hat{A}_{1}^{\alpha}(p)=\delta_{p, 0} Y^{\alpha}$. Imposing that condition, the Hilbert space of states invariant under all small gauge transformations becomes $\mathcal{H}_{\text {phys }}^{\prime}=$ $L^{2}\left(\mathbf{R}^{N_{\mathrm{P}}}, \mathrm{d} Y^{1} \cdots \mathrm{d} Y^{N_{\mathrm{P}}}\right) \otimes \mathcal{H}_{\text {Fermion }}^{\prime}$ where the zero charge sector in the fermion Fock space now is defined such that

$$
Q_{\mathrm{V}}^{\alpha}=\sum_{j=1}^{N_{\mathrm{F}}} \sum_{\sigma= \pm} n_{j, \sigma}^{\alpha} \hat{\rho}_{j, \sigma}(0)=0 \quad \forall \alpha=1, \ldots, N_{\mathrm{P}}
$$

on $\mathcal{H}_{\text {Fermion }}^{\prime}$ (again, these latter conditions come from the $(p=0)$-components of Gauss' law). Moreover, the Hamiltonian can again be written as $H=\frac{2 \pi}{L} \sum_{p \geq 0}: h_{p}:$.

For $p>0$ we can use the matrix notation introduced in Paragraph 6 and write $h_{p}=$ $\mathbf{c}^{\dagger} \cdot \mathbf{h c}$ where $\mathbf{h}=p \mathbf{1}+\frac{1}{p} \mathbf{Y}$ with

$$
\mathbf{Y}=\sum_{\alpha=1}^{N_{\mathrm{P}}} \frac{e^{\alpha} e^{\alpha}}{2 \pi} \mathbf{n}^{\alpha} \otimes\left(\mathbf{n}^{\alpha}\right)^{T}
$$

and $\left(\mathbf{n}^{\alpha}\right)^{T}=\left(n_{1,+}^{\alpha}, \ldots, n_{N_{\mathrm{F}},+}^{\alpha}, n_{1,-}^{\alpha}, \ldots, n_{N_{\mathrm{F}},-}^{\alpha}\right)$ etc. as before. We now show how to diagonalize these Hamiltonians, following the method explained in Paragraph 6: we compute $(\mathbf{q h})^{2}=p^{2} \mathbf{1}+\mathbf{Y}+\mathbf{q} \mathbf{Y q}$ where we used $\mathbf{Y q} \mathbf{Y}=0$ which follows from Eq. (2). We now observe that $\mathbf{Y}$ is a self-adjoint, real, non-negative matrix, and it therefore can be written as

$$
\mathbf{Y}=\sum_{j=1}^{N_{\mathrm{F}}} M_{j}^{2} \mathbf{e}_{j} \otimes \mathbf{e}_{j}^{T}
$$

\footnotetext{
${ }^{3}$ We believe that this assumption could be easily dropped.
} 
where the $\mathbf{e}_{j}$ are orthonormal and $M_{1}^{2} \geq M_{2}^{2} \geq \ldots \geq M_{N_{\mathrm{F}}}^{2}$. The $M_{j}^{2}$ and $\mathbf{e}_{j}$ can computed by diagonalizing the $N_{\mathrm{F}} \times N_{\mathrm{F}}$ matrix $\mathbf{Y}$. We also observe that the non-zero eigenvalues $M_{j}^{2}$ of the matrix $\mathbf{Y}$ are identical with the non-zero eigenvalues of the matrix $\mathbf{\Upsilon}=\left(\Upsilon^{\alpha \beta}\right)_{\alpha, \beta=1}^{N_{\mathrm{P}}}$ defined in Eq. (60). Thus only the $M_{j}^{2}$ with $j=1, \ldots, \operatorname{rank}(\mathbf{\Upsilon})=N_{\mathrm{P}}$ are non-zero. (To see this, note that if $\left(a_{j}\right)^{\alpha}$ are the components of an eigenvector of $\boldsymbol{\Upsilon}$ with non-zero eigenvalue $M_{j}^{2}$, then $\mathbf{f}_{j}=\sum_{\alpha} e^{\alpha}\left(a_{j}\right)^{\alpha} \mathbf{n}^{\alpha}$ is an eigenvector of the matrix $\mathbf{Y}$ with the same eigenvalue $M_{j}^{2}$. One can also check easily that the $\mathbf{f}_{j}$ span a space of dimension equal to the rank of the matrix $\Upsilon$ which we assumed to be equal to $N_{\mathrm{P}}$.)

Defining $\mathbf{e}_{N_{\mathrm{F}}+j}=\mathbf{q} \mathbf{e}_{j}$ we obtain a complete orthonormal basis in $\mathbf{R}^{2 N_{\mathrm{F}}}$ (orthogonality of the $\mathbf{e}_{j \leq N_{\mathrm{F}}}$ and $\mathbf{e}_{j>N_{\mathrm{F}}}$ again follows from Eq. (2)), and we can write

$$
(\mathbf{q h})^{2}=p^{2} \mathbf{1}+\sum_{j=1}^{N_{\mathrm{P}}} M_{j}^{2}\left(\mathbf{e}_{j} \otimes \mathbf{e}_{j}^{T}+\mathbf{e}_{N_{\mathrm{F}}+j} \otimes \mathbf{e}_{N_{\mathrm{F}}+j}^{T}\right) .
$$

Thus $\tilde{\mathbf{U}}$ Eq. (42) diagonalizes the matrix $(\mathbf{q h})^{2}$, and the eigenvalues of this latter matrix are $\omega_{j}^{2}=p^{2}+M_{j}^{2}$ where $M_{N_{\mathrm{F}}+j}^{2}=M_{j}^{2}$. It is then easy to check that all the Eqs. (43)-46) remain true also in the present case, and one finally obtains a representation of $h_{p}$ as in Eq. (47).

We now turn to $h_{0}$. After some computations we obtain,

$$
h_{0}=-\frac{1}{4 \pi}\left(\frac{L}{2 \pi}\right)^{2} \sum_{\alpha=1}^{N_{\mathrm{P}}} \frac{\partial^{2}}{\partial\left(\tilde{Y}^{\alpha}\right)^{2}}+\frac{1}{2} \sum_{\alpha, \beta=1}^{N_{\mathrm{P}}} \Upsilon^{\alpha \beta} \tilde{Y}^{\alpha} \tilde{Y}^{\beta}+\mathcal{C}
$$

where $\tilde{Y}^{\alpha}=Y^{\alpha}+\sum_{\beta}\left(\Upsilon^{-1}\right)^{\alpha \beta} Q_{\mathrm{A}}^{\beta}, Q_{\mathrm{A}}=\sum_{j, \sigma} \sigma n_{j, \sigma}^{\alpha} \hat{\rho}_{j, \sigma}(0)$, and

$$
\mathcal{C}=\frac{1}{2}\left(\sum_{j=1}^{N_{\mathrm{F}}} \sum_{\sigma= \pm} \hat{\rho}_{j, \sigma}(0)^{2}-\sum_{\alpha, \beta=1}^{N_{\mathrm{P}}} Q_{\mathrm{A}}^{\alpha}\left(\Upsilon^{-1}\right)^{\alpha \beta} Q_{\mathrm{A}}^{\beta}\right) .
$$

Note that $\tilde{Y}^{\alpha}, \mathcal{C}$ and $h_{0}$ all are invariant under the large gauge transformations

$$
\begin{aligned}
Y^{\alpha} & \rightarrow Y^{\alpha}-\frac{k^{\alpha}}{e^{\alpha}} \\
\hat{\rho}_{j, \sigma}(0) & \rightarrow \hat{\rho}_{j, \sigma}(0)+\sum_{\alpha=1}^{N_{\mathrm{P}}} \sigma n_{j, \sigma}^{\alpha} k^{\alpha}
\end{aligned}
$$

where $\mathbf{k}=\left(k^{1}, \ldots, k^{N_{\mathrm{P}}}\right) \in \mathbf{Z}^{N_{\mathrm{P}}}$.

Introducing $Z_{j}=\sum_{\alpha}\left(a_{j}\right)^{\alpha} \tilde{Y}^{\alpha}$ where $\left(a_{j}\right)^{\alpha}$ the components of the eigenvectors of the matrix $\Upsilon$ (cf. Eq. (61)) and $C_{j}^{(\dagger)}(0)=\stackrel{(-)}{+} \frac{L}{4 \pi}\left(\pi\left|M_{j}\right|\right)^{-1 / 2} \frac{\partial}{\partial Z_{j}}+\left(\pi\left|M_{j}\right|\right)^{1 / 2} Z_{j}$ we can write

$$
h_{0}=\sum_{j=1}^{N_{\mathrm{P}}} \frac{\left|M_{j}\right|}{2}\left(C_{j}(0)^{\dagger} C_{j}(0)+C_{j}(0) C_{j}(0)^{\dagger}\right)+\mathcal{C} .
$$


Again we can combine our results and write $H$ as in Eqs. (52)-(53), and we see explicitly that our model has a relativistic spectrum: we have $N_{\mathrm{P}}$ massive and $\left(N_{\mathrm{F}}-N_{\mathrm{P}}\right)$ massless bosons.

It is straightforward to extend the construction of the unitary operator $\mathcal{U}$ on $\mathcal{H}_{\text {Fermion }}^{\prime}$ diagonalizing all the $h_{p}$ and then check that $\varphi_{0}\left(Y^{1}, \ldots, Y^{N_{\mathrm{P}}}\right) \mathcal{U} \Omega_{\mathrm{F}} \in \mathcal{H}_{\text {phys }}^{\prime}$ with $\varphi_{0}=$ $\exp \left(-\frac{2 \pi^{2}}{L} \sum_{\alpha, \beta, \gamma}\left|M_{\gamma}\right|\left(a_{\gamma}\right)^{\alpha}\left(a_{\gamma}\right)^{\beta} Y^{\alpha} Y^{\beta}\right)$ (cf. Eq. (61)) is one groundstate of the model. One then can check that for any $\varphi \in L^{2}\left(\mathbf{R}^{N_{\mathrm{P}}}\right)$ which is an eigenstate of $h_{0}$ and any $\psi \in \mathcal{H}_{\text {Fermion }}^{\prime}$ which is a common eigenvector of all $h_{p}$, the state

$$
\varphi\left(\tilde{Y}^{1}, \ldots, \tilde{Y}^{N_{\mathrm{P}}}\right) \mathbf{R}^{\mathbf{m}} \Psi=\varphi\left(Y^{1}+\frac{k^{1}}{e^{1}}, \ldots, Y^{N_{\mathrm{P}}}+\frac{k^{N_{\mathrm{P}}}}{e^{N_{\mathrm{P}}}}\right) \mathbf{R}^{\sum_{\alpha} k^{\alpha} \mathbf{q n}^{\alpha}} \Psi
$$

with $\left(\mathbf{n}^{\alpha}\right)^{T} \cdot \mathbf{m}=1$ for all $\alpha$, is an eigenstate of $H$ with an eigenvalue of the form $E+c(\mathbf{m})$ where

$$
c(\mathbf{m})=\frac{2 \pi}{L}\left(\mathbf{m}^{2}-\sum_{j=1}^{N_{\mathrm{P}}}\left(\mathbf{e}_{j}^{T} \cdot \mathbf{q m}\right)^{2}\right)
$$

with $\mathbf{e}_{j}$ the orthonormal eigenvectors of the matrix $\mathbf{Y}$ in Eq. (63). With that one can check that the eigenstate of $H$ which also are invariant under the large gauge transformations are

$$
\begin{aligned}
& {[\Psi, \varphi]^{\left(\theta^{1}, \ldots, \theta^{N_{\mathrm{P}}}\right)}(Y)=\frac{1}{(2 \pi)^{N_{\mathrm{P}} / 2}} \sum_{\mathbf{k} \in \mathbf{Z}^{N_{\mathrm{P}}}} \mathrm{e}^{\mathrm{i} k^{1} \theta^{1}} \cdots \mathrm{e}^{\mathrm{i} k^{N_{\mathrm{P}} \theta^{N_{\mathrm{P}}}} \times}} \\
& \quad \times \varphi\left(Y^{1}+\frac{k^{1}}{e^{1}}, \ldots, Y^{N_{\mathrm{P}}}+\frac{k^{N_{\mathrm{P}}}}{e^{N_{\mathrm{P}}}}\right) \mathbf{R}^{k^{1} \mathbf{q n}^{1}} \cdots \mathbf{R}^{k^{N_{\mathrm{P}}} \mathbf{q n}^{N_{\mathrm{P}}}} \Psi
\end{aligned}
$$

where now we have $N_{\mathrm{P}}$ real $\theta$ parameters. Similarly as for $N_{\mathrm{P}}=1$ the physical Hilbert space $\mathcal{H}_{\text {phys }}$ of the model is spanned by the states $[\Psi, \varphi]^{(0, \ldots, 0)}(Y)$, and one needs to renormalize the inner product of these states, i.e. 'drop the infinite constant $\delta(0)^{N_{\mathrm{P}}}$ ', to get a proper inner product on $\mathcal{H}_{\text {phys }}$.

Acknowledgement: We thank M. Luescher for interesting discussions which prompted this work.

\section{References}

[1] R. Narayanan and H. Neuberger, Nucl. Phys. B443 (1995) 305; ibid. B 477 (1996) 521

[2] Sundermeyer K., Constrained Dynamics, Lecture Notes in Physics 169, Springer Verlag (1982) 
[3] E. Langmann and G.W. Semenoff, Phys. Lett. B341 (1994) 195

[4] H. Grosse, E. Langmann and E. Raschhofer, Ann. Phys. 253 (1997) 310

[5] D. Mattis and E. Lieb, J. Math. Phys. 6, (1965) 304

[6] N.S. Manton, Annals Phys. 159 (N.Y.) 220

[7] A.L. Carey and S.N.M. Ruijsenaars, Acta Appl. Mat. 10(1987) 1

[8] Araki H, Publ. Res. Inst. Math. Sci. Kyoto 6 (1970) 385 